\title{
A INFLUÊNCIA DA ÁREA DE ATUAÇÃO DO PROFESSOR NA INTERFACE DE OBJETOS DE APRENDIZAGEM POR ELE IDEALIZADOS
}

\author{
Diogo Israel Schwanck - PPGCC - FACIN - PUCRS \\ diogo.schwanck@acad.pucrs.br \\ Mára Lúcia Fernandes Carneiro - Instituto de Psicologia - UFRGS \\ mara.carneiro@ufrgs.br \\ Milene Selbach Silveira - PPGCC - FACIN - PUCRS \\ milene.silveira@pucrs.br
}

\begin{abstract}
Resumo: Com a ampla discussão acerca dos Objetos de Aprendizagem (OA) e sua inserção como elementos apoiadores nos processos de ensinar e de aprender, ampliamse as reflexões e preocupações quanto ao seu processo de desenvolvimento. Considerando a importância da interface desse recurso para o cumprimento de seus objetivos educacionais, este artigo apresenta os resultados de uma pesquisa relacionada às expectativas de professores proponentes de 44 OAs considerando as interfaces geradas pela equipe que os implementou. Os resultados da pesquisa apontam elementos que permitem estabelecer relações entre as áreas de conhecimento e a representação das interfaces criadas. São apresentados e discutidos exemplos contrastantes de projetos desenvolvidos.
\end{abstract}

Palavras-chave: objetos de aprendizagem, interface e interação, concepção.

Abstract: With the extensive discussion of Learning Objects (OA) and its insertion as supporting elements in the processes of teaching and learning, there are new concerns regarding their development process. Considering the importance of this resource interface for the fulfillment of their educational goals, in this paper we discuss the results of a research on the expectations of teachers proponents of 44 LOs regarding the interfaces created by the develop team. The research results indicate elements which establish relationships between areas of knowledge and the representation of the interfaces created. Are presented and discussed contrasting examples of projects developed.

Keywords: learning objects, interface and interaction, design.

\section{INTRODUÇÃO}

A discussão envolvendo o projeto, implementação e utilização de objetos de aprendizagem (OA) não é recente na comunidade de Informática na Educação. As primeiras pesquisas apontando esses recursos como elementos apoiadores dos processos de ensinar e de aprender datam do final da década de 1990. As pesquisas abordam diversos aspectos, tais como o desenvolvimento (Santos e Leite, 2010; Silva, Lima, et al., 2012; Serna, Castro e Botero, 2012), a avaliação (Vargo, et al., 2003; Reategui, Boff e Finco, 2008; Carneiro e Silveira, 2012; Silveira e Carneiro, 2012), a organização de repositórios (Araújo, 2003; Gluz e Vicari, 2010), entre outros.

Diversos são, também, os conceitos utilizados para se definir um OA (Hodgins, 2000; Wiley, 2000; Vargo, et al., 2003; Tarouco, Fabre e Tamusiunas, 2003; Johnson, 2003; Kay e Knaack, 2008). Entretanto, independente do conceito utilizado para sua definição, consideramos fundamental - para a concepção de um OA (e para que, 
portanto, um determinado material/recurso didático possa ser assim denominado) - que este contemple as seguintes características (Carneiro e Silveira, 2012):

- Ser digital;

- Explicitar claramente um objetivo pedagógico;

- Disponibilizar ajuda à navegação;

- Oferecer interatividade;

- Prever ações que incentivem a interação;

- Ser autocontido;

- Disponibilizar orientações de uso para outros professores.

E o ponto que une todas estas características é a interface do objeto. A interface de um sistema interativo, neste caso, um OA, compreende, segundo Moran (1981), todos os aspectos de um sistema com o qual o usuário tem contato durante a interação. Segundo Barbosa e da Silva (2010), este pode ser um contato físico (por meio de hardware e software) ou um contato conceitual (interpretação do usuário sobre o que ele percebe a partir do contato físico). Estes autores destacam que a interface com o usuário determina os processos de interação possíveis, à medida que determina o que ele pode falar ou fazer, de que maneira e em que ordem.

Quanto às características de um OA, anteriormente listadas, ser digital e oferecer interatividade e incentivo a interação implicam no contato físico e compreensão deste pelo contato conceitual. E os processos de interação possíveis são determinados por meio da interface do objeto, que tem foco em um determinado assunto/conteúdo (e consegue trabalhá-lo de forma independente), e são explicitados por meio da apresentação de seu objetivo pedagógico, de ajuda à navegação e de orientações para uso.

Considerando-se, então, a interface, o elo que liga estas diferentes características, será o objeto de análise e reflexão do presente trabalho, tentando se responder a seguinte questão de pesquisa: "O perfil do professor - considerando sua área de conhecimento- influencia a interface do OA por ele idealizado?”. Sendo assim, propomos duas análises distintas: (1) quantitativa comparativa coletiva, baseada nas áreas de conhecimentos para o qual os OA foram projetados e (2) qualitativa comparativa individual, utilizando interfaces com características semelhantes e distintas.

Para este estudo, considerou-se a metodologia para construção de OAs seguidas por uma equipe que trabalha no apoio à construção de OAs por professores de ensino superior de uma determinada Universidade. Neste contexto, os professores planejam, junto a essa equipe, seus OAs, os quais são - do ponto de vista computacional implementados por essa equipe. Em uma das etapas da concepção do objeto, os professores devem responder a um questionário, que - dentre outros itens - contém as características esperadas da interface que será projetada. É a partir da análise destes dados específicos, advindos das respostas a 44 questionários por professores produtores de OA, que são apresentadas as considerações integrantes deste artigo.

O artigo está organizado da seguinte forma: a seção 2 apresenta o contexto do estudo e a metodologia usada para coleta de dados; a seção 3, apresenta a análise dos dados e as respectivas discussões e achados; e, a seção 4 apresenta as considerações finais seguidas pelas referências nele utilizadas. 


\section{CONTEXTO DO ESTUDO}

O Núcleo de Apoio Pedagógico à Educação a Distância (NAPEAD) está vinculado a Universidade Federal do Rio Grande do Sul (UFRGS), é coordenado por professores com experiência em EAD e Informática na Educação e reúne uma equipe interdisciplinar de profissionais e bolsistas que têm por objetivo apoiar a produção de OAs de forma colaborativa, fazendo com que o professor proponente um parceiro ativo no processo de concepção, modelagem, implementação, teste e avaliação dos seus objetos de aprendizagem. Atualmente a equipe conta, além de sua coordenação (professor da área de Design), com uma pedagoga, um programador visual e oito bolsistas com experiência na produção de vídeos, animações e hipertextos.

A partir da divulgação de edital específico, os professores da universidade podem submeter seus projetos para desenvolvimento de um ou mais OAs. Após a submissão, a equipe gestora do núcleo realiza uma seleção, baseada no detalhamento e na aplicabilidade do projeto apresentado pelo professor. Com a aprovação, inicia o processo de construção do OA.

Para esta construção, a partir de 2011, foi adotada a metodologia INTERAD Interfaces Interativas Digitais Aplicadas à Educação (Figura 1). Esta adoção teve como objetivo qualificar o trabalho e aprimorar o processo de produção de objetos de aprendizagem pelo núcleo.

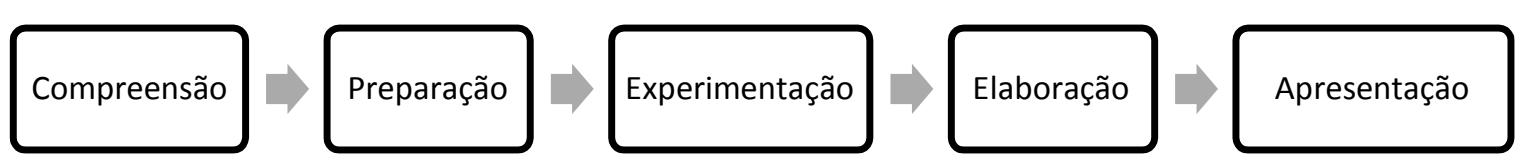

Figura 1 - Metodologia INTERAD

A metodologia INTERAD orienta o desenvolvimento de interfaces para materiais educacionais digitais, e está dividida em cinco etapas (Passos, 2011):

- Compreensão: busca de subsídios para o projeto, levantamento de informações relativas ao tema, objetivos pedagógicos, necessidades do usuário, público-alvo, filosofia da instituição, conceito visual do material e contexto de aplicação.

- Preparação: descrição de elementos e funcionalidades necessárias para atingir os objetivos pedagógicos propostos; definição de equipe; seleção de recursos interativos e de mídia apropriada; levantamento de conteúdo;

- Experimentação: organização do conteúdo conforme a proposta interativa; design de interação; desenho dos fluxos de tarefa;

- Elaboração: projeto gráfico; definição da hierarquia da informação; composição dos elementos gráficos através de modularização; agrupamento do conteúdo;

- Apresentação: design visual; definição dos atributos gráficos e apresentação de textos e imagens.

Assim, para apoiar a equipe na compreensão e detalhamento do projeto proposto pelo professor foi adotado um formulário, baseado na proposta de Passos (2011). Uma das seções do formulário referia-se às características esperadas da interface que seria projetada para o objeto de aprendizagem. Como forma de facilitar este levantamento de características, foi adotada a escala de diferencial semântico, técnica criada por Osgood, Suci e Tannenbaum (1957), com o objetivo de avaliar o significado que as pessoas 
atribuem a conceitos ou objetos. A técnica envolve pares de adjetivos bipolares, usados como escala de medida, baseando-se na ideia de que os adjetivos selecionados podem ser usados para medir os sentimentos associados àquele conceito. Esta escala mostra que "universalmente as palavras que constituem a matéria-prima do nosso pensamento já trazem no seu significado conteúdos emocionais” (Lane, 1995, p. 58-59).

Em sua proposta original, Passos (2011) utilizou essa técnica para identificar as características da interface, segundo a expectativa de seus proponentes, a partir da seleção, por estes, de atributos de um conjunto de 15 pares de adjetivos bipolares, conforme demonstrado na Figura 2.

\begin{tabular}{|c|c|}
\hline Tradicional & Inovadora \\
\hline Humana & Tecnológica \\
\hline Geométrica & Orgânica \\
\hline Simples & Sofisticada \\
\hline Consolidada & Lançamento \\
\hline Modesta & Imponente \\
\hline Adulta & Infantil \\
\hline Divertida & Agressiva \\
\hline Lúdica & Séria \\
\hline Dinâmica & Estável \\
\hline Regional & Internacional \\
\hline Popular & Especializada \\
\hline Metafórica & Alternativa \\
\hline Minimalista & Rebuscada \\
\hline Feminina & Masculina \\
\hline
\end{tabular}

Figura 2 - Quadro de diferencial semântico com as expectativas do proponente FONTE: Passos (2011)

O quadro utilizado pela equipe do Núcleo foi adaptado a partir da proposta de Passos, adotando uma Escala de Likert para avaliar os registros e permitir uma análise dos resultados. A Figura 3 apresenta os adjetivos selecionados para descrição das características esperadas do objeto de aprendizagem pelos professores proponentes.

\begin{tabular}{r|l|l|l|l|l|l}
\hline & $\mathbf{1}$ & $\mathbf{2}$ & $\mathbf{3}$ & $\mathbf{4}$ & $\mathbf{5}$ & \\
\hline tecnológica & & & & & & humana \\
\hline simples & & & & & & sofisticada \\
\hline objetiva & & & & & & lúdica \\
\hline especializada & & & & & & regional \\
\hline minimalista & & & & & & popular \\
\hline
\end{tabular}

Figura 3 - Escala de Diferencial Semântico adaptada pelo núcleo de desenvolvimento de OAs

Como base para a análise efetuada neste trabalho, utilizou-se dados referentes a um edital lançado em 2012. Por meio deste edital, disponibilizou-se a execução de 60 projetos de OAs, divididos em duas fases: Fase 1, contemplando 20 projetos, e Fase 2, contemplando os demais 40 projetos.

O formulário descrito anteriormente foi implementado durante a Fase 2 desse edital, por isso foi preenchido por apenas 44 professores. O grupo formado era composto por professores de diversas áreas de conhecimento ${ }^{1}$ - Ciências Exatas e da Terra, Ciências Biológicas, Engenharias, Ciências da Saúde, Ciências Agrárias, Ciências Sociais Aplicadas, Ciências Humanas e Linguística, Letras e Artes - e a

\footnotetext{
${ }^{1}$ Conforme distribuição do Conselho Nacional de Desenvolvimento Científico e Tecnológico (CNPq).

Disponível em <http://memoria.cnpq.br/areasconhecimento/index.htm>. Acesso em 07/05/2014.
} 
distribuição dos participantes dessa pesquisa dentro dessas áreas está representada na Figura 4.

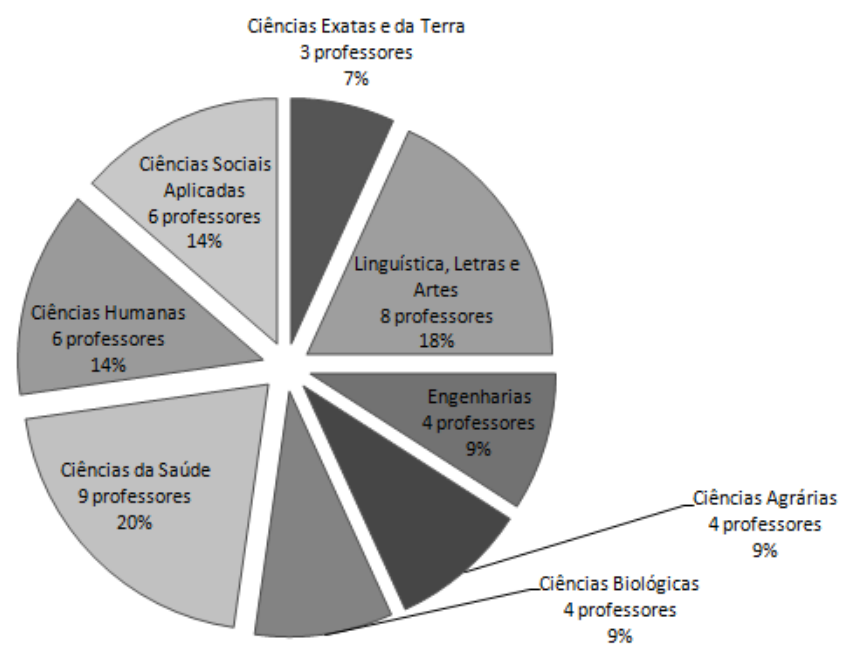

Figura 4 - Número de respondentes por área de conhecimento

Relacionando especificamente as características esperadas da interface, via coleta de dados do quadro de diferencial semântico, a Tabela 1 resume o levantamento dos dados, apresentando as modas ${ }^{2}$ das respostas fornecidas em cada uma das categorias de adjetivos bipolares, de acordo com a área de atuação dos professores.

Tabela 1 - Resultados Obtidos

\begin{tabular}{|c|c|c|c|c|c|c|}
\hline & $\begin{array}{c}\text { Tecnológica (1) } \\
\text { humana (5) }\end{array}$ & $\begin{array}{c}\text { Simples (1) } \\
\text { Sofisticada(5) }\end{array}$ & $\begin{array}{l}\text { Objetiva (1) } \\
\text { Lúdica(5) }\end{array}$ & $\begin{array}{c}\text { Internacional (1) } \\
\text { Regional (5) }\end{array}$ & $\begin{array}{c}\text { Especializada(1) } \\
\text { Popular(5) }\end{array}$ & $\begin{array}{l}\text { Minimalista(1) } \\
\text { Rebuscada(5) }\end{array}$ \\
\hline Ciências Agrárias & 3 & 2 & 2,5 & 3 & 1 & 2 \\
\hline Ciências Biológicas & 4 & 2 & 4 & 1 & 2,5 & 3 \\
\hline Ciências da Saúde & 3 & 1 & 1 & 3 & 1 & 3 \\
\hline $\begin{array}{c}\text { Ciências Exatas e } \\
\text { da Terra }\end{array}$ & 1 & 2 & 3 & 3 & 2 & 2 \\
\hline Ciências Humanas & 5 & 1 & 1 & 3 & 2 & 2 \\
\hline $\begin{array}{l}\text { Ciências Sociais } \\
\text { Aplicadas }\end{array}$ & 5 & 3 & 2 & 3 & 2 & 3 \\
\hline Engenharias & 1 & 3 & 2 & 1 & 1 & 3 \\
\hline $\begin{array}{c}\text { Lingüística, Letras } \\
\text { e Artes }\end{array}$ & 3 & 1 & 1 & 1 & 3 & 2 \\
\hline
\end{tabular}

Destacam-se, ainda na Tabela 1, as categorias consideradas amodais, das quais se apresentou, então, a mediana dentre os dados obtidos, conforme dados destacados.

\section{ANÁLISE DOS DADOS}

Os dados coletados nessa pesquisa foram analisados relacionando, inicialmente, as áreas de conhecimento e, posteriormente, comparando algumas das interfaces construídas com o uso das informações dadas à equipe de desenvolvimento através do formulário citado anteriormente.

\footnotetext{
${ }^{2}$ Tendo em vista o tipo de dados a serem analisados (dados ordinais), não é recomendada a utilização da média, visto que a Escala de Likert pode sofrer tendência na existência de dados extremos (outliers).
} 


\subsection{Comparando as áreas de conhecimento}

A partir das respostas ao questionário apresentadas pelos docentes, realizou-se uma análise quantitativa comparativa coletiva, baseada na avaliação geral entre os dados e, posteriormente, relacionando cada par de categorias quanto à diferença entre os graus extremos apresentados, através do teste de Kruskal-Wallis ${ }^{3}$ (KW).

Através do resultado do teste de $\mathrm{KW}$, temos evidência de diferença significativa na categoria Tecnológica-Humana $(\mathrm{p}<0,01)$, onde se encontram assemelhadas as áreas de Ciências Exatas e da Terra e Engenharias, com predominância da característica tecnológica, díspares da área de Ciências Humanas, a qual ressalta a característica humana, conforme pode ser observado nos gráficos de diferencial semântico apresentados nas Figuras 5 e 6. Nas demais situações analisadas (entre diferentes áreas e categorias de características), não há indícios de diferença significativa.

\begin{tabular}{|c|c|c|c|c|c|}
\hline & $\begin{array}{lllll}1 & 2 & 3 & 4 & 5\end{array}$ & (a) & & $\begin{array}{lllll}1 & 2 & 3 & 4 & 5\end{array}$ & (b) \\
\hline Tecnológica & $a$ & Humana & Tecnológica & & Humana \\
\hline Simples & & Sofisticada & Simples & & Sofisticada \\
\hline Objetiva & ๑ & Lúdica & Objetiva & & Lúdica \\
\hline Internacional & & Regional & Internacional & i & Regional \\
\hline Especializada & i & Popular & Especializada & & Popular \\
\hline Minimalista & - & Rebuscada & Minimalista & 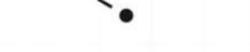 & Rebuscada \\
\hline
\end{tabular}

Figura 5 - Gráfico de diferencial semântico das áreas de Ciências Exatas e da Terra (a) e Engenharias (b)

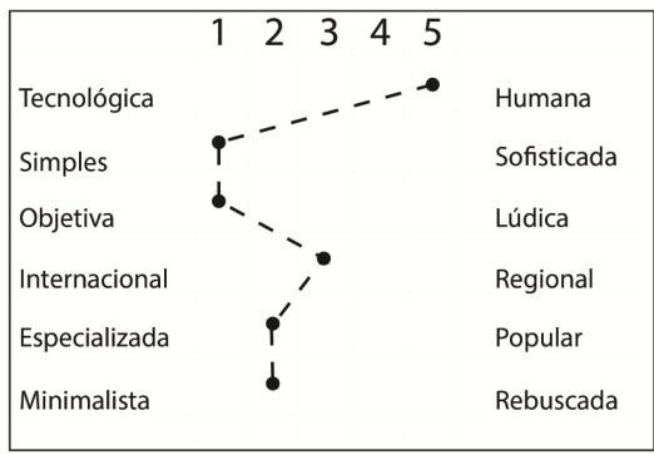

Figura 6 - Gráfico de diferencial semântico da área de Ciências Humanas

Ainda sobre os gráficos das Figuras 5 e 6, podemos perceber que, apesar das diferenças ressaltadas na análise estatística, ainda podemos citar semelhanças entre estas áreas contrastantes, como a relação entre o par de adjetivos Especializada-Popular, o qual é indicado fortemente pela área das Engenharias e de forma moderada pelas áreas de Ciências Exatas e da Terra e Ciências Humanas.

\subsection{Apresentando e relacionando Objetos de Aprendizagem}

A fim de exemplificar a análise realizada, neste item apresentam-se três casos de objetos produzidos e descreve-se a análise qualitativa comparativa individual, levando em conta

\footnotetext{
${ }^{3}$ Teste estatístico para avaliação de diferença entre amostras independentes.
} 
as características ressaltadas pelos professores proponentes relacionadas às interfaces produzidas, possibilitando, assim, a análise de diferenciação e aproximação dos mesmos. Foram selecionados OAs que apresentaram nas respostas de seus proponentes características consideradas extremistas, isto é, ao menos duas respostas "1" ou "5", e, simultaneamente, divergentes.

O OA "Luz, Câmera e História" (Figura 7) foi desenvolvido a partir da proposta de uma docente da área de Ciências Humanas com o objetivo de "apresentar alternativas, propiciar desafios e reflexões acerca das possibilidades de produção $e$ utilização de vídeos no ensino de História" para alunos de Estágio Docente da Instituição vinculada, e, ao responder o questionário de diferencial semântico, as respostas da proponente destacavam as seguintes características: Humana e Simples.
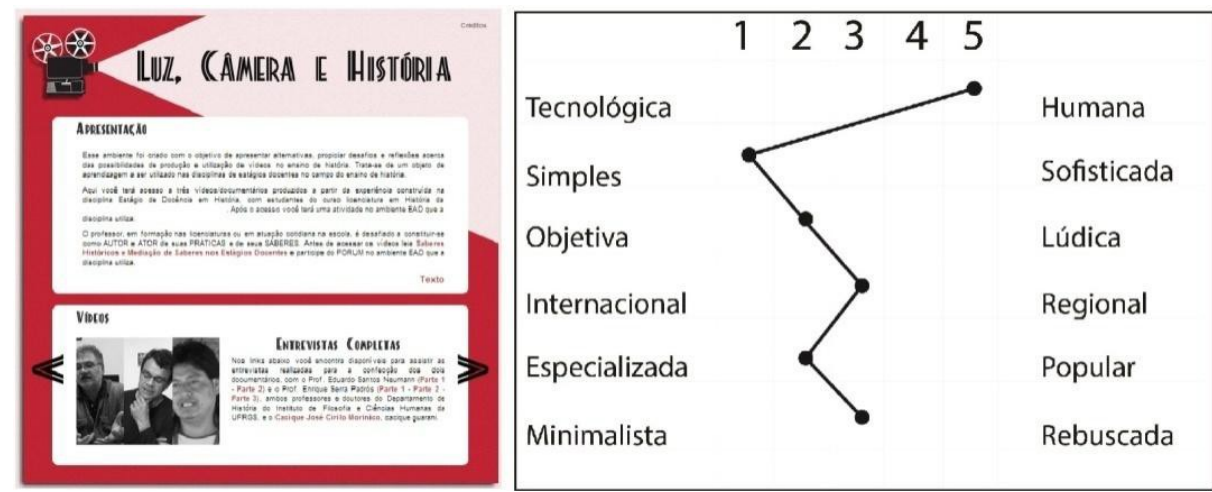

Figura 7 - Interface do OA "Luz, Câmera e História" e seu gráfico de diferencial semântico

Podemos identificar, através da análise visual, que a característica "Simples" foi contemplada através o detalhamento das informações relacionadas tanto ao objeto quanto ao conceito a ser apresentado e em uma interface limpa e autoexplicativa. Isso se repete em demais casos construídos e disponíveis no repositório do núcleo. Ainda sobre essa interface, percebe-se que a mesma se preocupa com a identidade humana, no uso de imagens e signos alusivos à temática do objeto, atendendo, assim, a característica "Humana" ressaltada pela proponente.

Contrastante ao "Luz, Câmera e História", apresentamos o OA "Diagramas de Ellingham: Óxidos" (Figura 8), que foi baseado no projeto de uma professora da área de Engenharia, com o objetivo de possibilitar a comparação entre os gráfico de temperatura versus energia livre dentre as reações selecionadas.Através dos resultados obtidos pelo questionário respondido pela docente, foram citadas as seguintes características: Tecnológica, Internacional e Especializada.

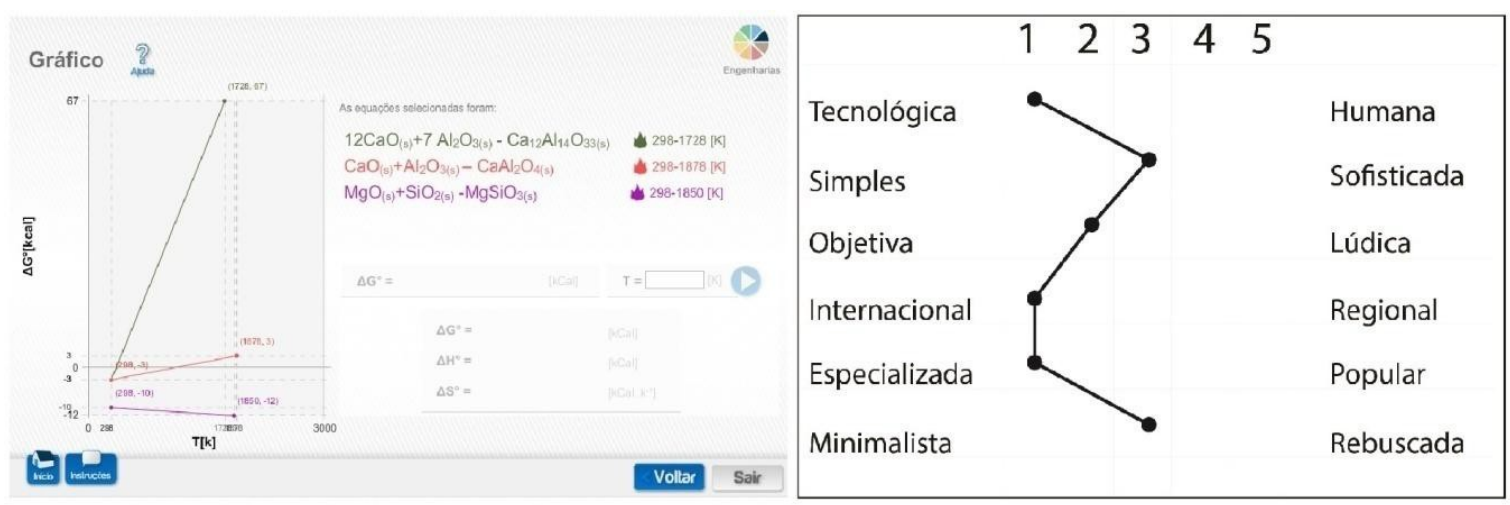

Figura 8 - Interface do OA "Diagramas de Ellingham" e seu gráfico de diferencial semântico 
A característica "Tecnológica" pode ser identificada através da construção dos gráficos, após a escolha das reações a serem comparadas, e por conta da interatividade proporcionada, ao passo que o usuário tem a possibilidade de escolher um valor referência para analisar dentre as reações escolhidas. Já as características de “especializado" e “internacional” podem ser verificadas pela utilização de nomenclaturas e notações específicas dentre as reações e os resultados apresentadas e, ainda, dada a utilização de notação universal utilizada nas legendas e reações.

Aqui caracterizado como um exemplo mais atípico, o OA "SUSCity" (Figura 9) foi construído sob responsabilidade de uma docente da área das Ciências da Saúde, para uso em ambiente virtual que apresenta os paradigmas propostos pelo Sistema Único de Saúde (SUS), quanto à distribuição dos serviços de saúde e utilização de indicadores para o planejamento de ações, relacionados a um cenário construído pelo usuário dentro de uma comunidade fictícia. Para tanto, o objeto foi concebido em forma de um jogo baseado em uma cidade virtual. Realizado o preenchimento do questionário, citaram-se as seguintes características de interface: Humana, Sofisticada, Lúdica, Regional e Rebuscada.

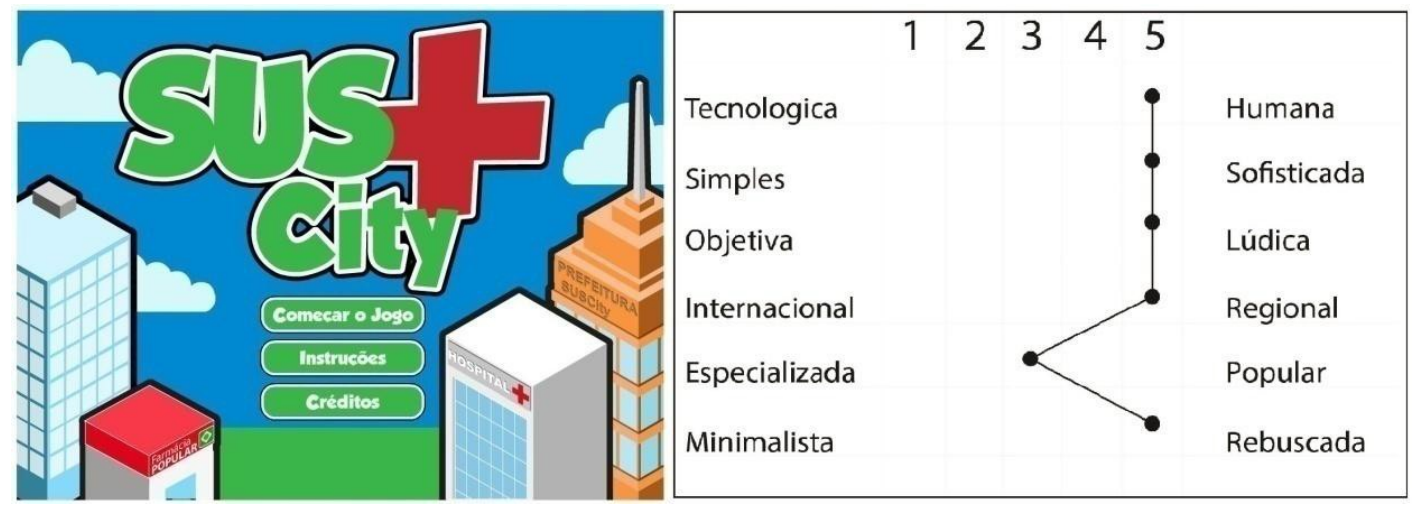

Figura 9 - Interface do Objeto de Aprendizagem SUSCity e seu gráfico de diferencial semântico

Podemos perceber, inicialmente, que por sua concepção em forma de jogo baseado em uma cidade virtual com riqueza de detalhes, garantem-se o atendimento de quatro características relacionadas pela docente para a interface: Humana, Sofisticada, Lúdica e Rebuscada. Para tentar atender a característica Regional, houve a inclusão de diferentes tipos de edificações (casas, prédios, barracos, prédios comerciais, etc.) para que o usuário pudesse simular da forma mais real possível o contexto escolhido.

A análise realizada, através da comparação entre as interfaces produzidas e as características esperadas pelo professor proponente, registradas nos quadros de diferencial semântico, mesmo que ainda inicial, aponta a importância da participação da equipe de design no processo de produção de OAs. Como, em geral, essa implementação é realizada pela equipe de bolsistas, o levantamento parece apontar a demanda por um estudo mais aprofundado do tema/conteúdo do OA, para que a interface utilize signos representativos do cotidiano para a representação de interfaces humanas coerentes com as expectativas do professor.

\section{CONSIDERAÇÕES FINAIS}

A interface de um OA é vital para sua caracterização, uma vez que ela auxilia no entendimento da mensagem desejada por aquele que o desenvolveu e, assim sendo, o OA possa atingir seu objetivo pedagógico para o qual foi criado. 
Através das análises realizadas e a questão levantada acerca das áreas de conhecimento e do perfil do professor, pode-se perceber indícios de tendência entre três áreas de conhecimento. Como indícios, esses resultados motivam a seguir essa investigação - voltada às áreas de conhecimento - e, para torná-los mais consistentes, uma nova etapa de coleta de dados está em andamento. Este fato pode auxiliar a emergir novos pontos relacionados aos docentes e a área de atuação desses, que devem ser considerados na construção da interface de um objeto de aprendizagem para além das demandas já levantadas com o apoio dos quadros de diferencial semântico.

Neste trabalho apresentamos alguns dos objetos que mais contrastam em suas características e, através dessa análise cabe, também, um novo questionamento: qual a percepção do proponente a respeito da interface construída? A concretização - na interface do OA - das características elencadas realmente constitui o que era esperado por ele? Este ponto constitui, também, um dos próximos passos desta pesquisa, a análise da percepção do professor proponente sobre resultado final dessa caminhada rumo a um novo recurso para a ação docente.

\section{Referências}

ARAÚJO, M. D. Educação à Distância e a WEB Semântica: modelagem ontológica de materiais e objetos de aprendizagem para a plataforma COL. São Paulo: Escola Politécnica de São Paulo, Departamento de Engenharia e Sistemas Digitais, 2003. 178 p. Tese de doutorado.

BARBOSA, S. D. J.; SILVA, B. S. D. Interação Humano-Computador. Rio de Janeiro: Elsevier, 2010.

CARNEIRO, M. L. F.; SILVEIRA, M. S. Objetos de Aprendizagem Sob o Ponto de Vista dos Alunos: Um Estudo de Caso. Revista Novas Tecnologias em Educação, Porto Alegre, v. 10, n. 3, dez. 2012.

GLUZ, J. C.; VICARI, R. M. MILOS: Infraestrutura de Agentes para Suporte a Objetos de Aprendizagem OBAA. Anais do Simpósio Brasileiro de Informática na Educação. João Pessoa: Sociedade Brasileira de Computação. 2010.

HODGINS, H. W. Into the Future - A Vision Paper. Comission on Technology \& Adult Learning. Alexandrina, p. 45. 2000.

JOHNSON, L. F. Elusive Vision: Challenges Impeding the Learning Object Economy. San Francisco: Macromedia, Incorporated, 2003.

KAY, R. H.; KNAACK, L. A formative analysis of individual differences in the effectiveness of learning objects in secondary school. Computers \& Education, Londres, v. 51, n. 1, 2008. p. 1304-1320.

LANE, S. T. M. A mediação emocional na construção do psiquismo humano. In: LANE, S. T. M.; SAWAIA, B. B. Novas Veredas da Psicologia Social. São Paulo: Brasiliense, 1995.

MORAN, T. P. The Command Language Grammar: a representation for the user interface of interactive computer systems. International Journal of Man-Machine Studies, v. 15, n. 1, p. 3-50, jul/1981. 
OSGOOD, C. E.; SUCI, G.; TANNENBAUM, P. The measurement of meaning. Urbana: University of Illinois Press, 1957.

PASSOS, P. C. S. J. Interad: uma metodologia para design de interface de materiais educacionais digitais. Porto Alegre: Universidade Federal do Rio Grande do Sul, Faculdade de Educação, Programa de Pós-Graduação em Informática na Educação, 2011. Dissertação de mestrado.

REATEGUI, E.; BOFF, E.; FINCO, M. D. Proposta de Diretrizes para Avaliação de Objetos de Aprendizagem Considerando Aspectos Pedagógicos e Técnicos. RENOTE: Revista Novas Tecnologias em Educação, Porto Alegre, v. 8, n. 3, 2008.

SANTOS, P. K. D.; LEITE, L. L. O desenvolvimento de Objetos de Aprendizagem para Educação a Distância ancorados pelas Dimensões da Educação. Revista Educação por Escrito - PUCRS, Porto Alegre, v. 1, n. 1, p. 76-86, 2010.

SERNA, E. M.; CASTRO, C. A. C.; BOTERO, R. T. SEDLO: Software Engeneering for Developing Learning Objects. EATIS 2012 Conference Proceedings. Valencia: ACM. 2012. p. 347-353.

SILVA, T. R. et al. O uso de ferramentas pedagógicas no desenvolvimento de Objetos de Aprendizagem. Workshop de Desafios da Computação Aplicada à Educação (DesafIE). Curitiba: Sociedade Brasileira de Computação. 2012. p. 188-197.

SILVEIRA, M. S.; CARNEIRO, M. L. F. Diretrizes para a Avaliação de Usabilidade de Objetos de Aprendizagem. Anais do Simpósio Brasileiro de Informática na Educação, v. 23, n. 1, 2012.

TAROUCO, L. M. R.; FABRE, M.-C. J.; TAMUSIUNAS, F. Reusabilidade de objetos educacionais. Revista Novas Tecnologias em Educação, Porto Alegre, v. 1, n. 1, 2003.

VARGO, J. A. et al. Learning object evaluation: Computer-mediated collaboration and inter-rater reliability. International Journal of Computers and Applications, Anaheim, 2003. p. 198-205.

WILEY, D. A. Learning object design and sequencing theory. Provo: Brigham Young University, 2000. Tese de doutorado. 